\title{
MORITA EQUIVALENCE OF SEMIGROUPS REVISITED: FIRM SEMIGROUPS
}

\author{
VALDIS LAAN, LÁSZLÓ MÁRKI, AND ÜLO REIMAA
}

\begin{abstract}
We define firm semigroups and firm acts as non-additive analogues of firm rings and firm modules. Using the categories of firm acts we develop Morita theory for firm semigroups. We show that equivalence functors between categories of firm acts over two firm semigroups have to be tensor multiplication functors. Our main result states that the categories of firm right acts over two firm semigroups are equivalent if and only if these semigroups are strongly Morita equivalent, which means that they are contained in a unitary Morita context with bijective mappings.

We also investigate other categories of acts which have been used earlier to develop Morita equivalence. The main tool in our work is adjoint functors. We prove that over firm semigroups all the considered categories are equivalent to the category of firm acts.

All this suggests that firm semigroups and firm acts are the natural environment to study Morita equivalence of semigroups.
\end{abstract}

\section{INTRODUCTION}

A theory of Morita equivalence was carried over from unital rings to monoids independently by Banaschewski [6] and Knauer [21] in the early seventies but their results have not really been taken up. In the eighties, Morita equivalence was extended to much wider classes of rings [1], [5], [17], no longer requiring the existence of an identity element. To construct a useful theory of Morita equivalence in the non-unital case, one had to restrict both the class of rings and the class of modules to be considered. Based on the development in [5], Talwar found a viable approach to Morita equivalence for semigroups without identity but with certain idempotents called local units [35], showing also the relevance of Morita equivalence in the structure theory of semigroups. He also extended some results to factorisable semigroups (those in which every element decomposes as a product) [36], [37]. Subsequently, Chen and Shum [9] as well as Neklyudova [29], [30] contributed to the theory. These authors started from different approaches to Morita equivalence of rings without identity: Talwar's development follows that of Ánh and Márki [5],

Date: 26.04 .2017$.

2010 Mathematics Subject Classification. 20M30, 20M50.

Key words and phrases. Firm semigroup, firm act, unitary act, Morita equivalence, strong Morita equivalence, adjoint functors, localization, colocalization.

Research of the first and third named authors was partially supported by the Estonian Institutional Research Project IUT20-57, research of the first named author was also partially supported by the Estonian Research Council's grant PUT1519, research of the second named author was partially supported by the Hungarian National Research, Development and Innovation Office, NKFIH, no. K119934. Mutual visits of the authors were made possible by the exchange agreement between the Estonian and the Hungarian Academies of Sciences. 
Chen and Shum use techniques similar to those of García and Simón [17], whereas Neklyudova goes back to the more restrictive development of Abrams [1].

A decisive step was made by Lawson [27] in 2011. He considered the class of semigroups with local units, defined in the same way as by Talwar [35]. However, instead of Talwar's fixed acts he considered closed acts - these are easier to get around with than fixed acts, and Lawson proved that these two kinds of acts coincide over semigroups with local units. He also filled in two gaps in Talwar's treatment. From our point of view, the main result in Lawson's work is the fact that, for semigroups with local units, every Morita equivalence is strong in the sense that it comes from a unitary Morita context with surjective mappings. Lawson [27] as well as Laan and Márki [23] give various structural characterisations of Morita equivalence for semigroups with local units. By all this one can say that we have a satisfactory theory of Morita equivalence for semigroups with local units. Based on these works, structural aspects of Morita equivalence (including Morita invariant properties) have been considered in [3], [4], [14], [24], [33], [34].

Attempts have been made also to extend the theory beyond semigroups with local units. At first, Laan [22] noticed that strong Morita equivalence can occur only between factorisable semigroups - thus expressing in precise terms what Lawson formulated roughly at the same time in [27] as "current thinking is that factorisable semigroups form the largest class of semigroups for which a useful Morita theory can be developed'. The main questions were whether it is true in more general classes than semigroups with local units that every Morita equivalence is strong; if not, whether equivalence of appropriate categories of right acts implies equivalence of the corresponding categories of left acts. In particular, whether these are true for factorisable semigroups in general.

Work in this direction has been inspired by papers on Morita equivalence on nonunital rings by the Spanish school, mainly by Marín and his coauthors, continuing the development started by García and Simón [17]. Laan and Márki [25] considered so-called fair semigroups - this class corresponds to the class of xst-rings considered by García and Marín [15], based on previous work by Xu, Shum, and Turner-Smith [39] (whence the name of these rings). Among many other results, it is shown in [25] that every finite monogenic semigroup is Morita equivalent to its group part, thus we have examples of non-factorisable semigroups which are Morita equivalent to groups, and strong Morita equivalence is impossible between them.

In the main line of the present paper we consider the same class of acts as was done in [27], [23] and [25]. There they were called 'closed acts' - however, here we call them 'firm acts'. Namely, these acts are exactly the non-additive analogues of modules called 'firm modules' by Quillen [31], used later also in many papers by Marín. (Notice the strange coincidence with the fact that Lawson kept Talwar's notation FAct for the category of these acts, referring to the French word 'fermé' for 'closed'.) We call a semigroup 'firm' if it is a firm act over itself. The main result of our paper is that two firm semigroups are strongly Morita equivalent if and only if the categories of firm right acts over these semigroups are equivalent. We also consider other categories of acts used for building Morita theory by other authors, as well as categories of acts which correspond to categories of modules used by García and Marín [16], with the aim of clarifying the relations between these categories. Our main tool is the usage of adjunctions between various categories of acts. In our eyes, the results in the present paper are convincing enough to claim 
that firm semigroups and firm acts are the natural environment to study Morita equivalence of acts.

In Section 2 we introduce firm acts and semigroups and list some basic facts about them. In Section 3 we study in detail the adjunctions that appear between different act categories. We show that fixed and firm acts are the same over firm semigroups and that three natural choices for act categories used in Morita theory are all equivalent for firm semigroups, so from the point of view of Morita theory it makes no difference which of them one uses. Section 4 is devoted to giving a description of equivalence functors between categories of firm acts over firm semigroups. It turns out that even in this general situation such functors have to be tensor multiplication functors. Section 5 contains an overview of some results in the Morita theory of semigroups and the proof of our main result.

Acknowledgement. Thanks are due to Peter Vámos for providing a copy of notes taken at D. Quillen's lecture at the University of Exeter on February 8, 1996.

\section{Firm ACTS AND SEMigroups}

We start by recalling some definitions. A semigroup $S$ is called factorisable if every element of $S$ is a product of two elements. We say that an element $s$ of a semigroup has a weak right local unit $u$ (weak left local unit $v$ ) if $s u=s$ $(v s=s)$. A semigroup has weak local units if each of its elements has both a weak right and a weak left local unit, and local units if the elements $u, v$ above can always be chosen to be idempotent.

We say that a semigroup $S$ has common weak right local units if for every $s, t \in S$ there exists $u \in S$ such that $s=s u$ and $t=t u$. Semigroups with common weak left local units are defined dually. A semigroup has common weak local units if it has common weak right local units and common weak left local units.

Let $S$ and $T$ be semigroups. We use the notation $\operatorname{Act}_{S}\left({ }_{S}\right.$ Act, $\left.{ }_{S} \mathrm{Act}_{T}\right)$ for the category of all right $S$-acts (left $S$-acts, $(S, T)$-biacts) where morphisms are right $S$-act homomorphisms (left $S$-act homomorphisms, $(S, T)$-biact homomorphisms). A right $S$-act $A_{S}$ is called unitary if $A S=A$. We denote the category of all

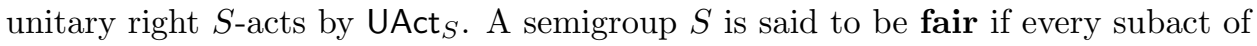
a unitary right $S$-act and every subact of a unitary left $S$-act is unitary, see [25].

Definition 2.1. We say that a right $S$-act $A_{S}$ is firm if the mapping

$$
\mu_{A}: A \otimes S \rightarrow A, a \otimes s \mapsto a s
$$

is bijective. A semigroup $S$ is called firm if it is firm as a right (or, equivalently, left) $S$-act.

The category of all firm right $S$-acts is denoted by FAct $_{S}$.

Obviously, $A_{S}$ is unitary if and only if the mapping $\mu_{A}$ is surjective. Hence, for any semigroup $S$, FAct $_{S}$ is a subcategory of $\mathrm{UAct}_{S}$. Also, a semigroup $S$ is factorisable if and only if $\mu_{S}$ is surjective.

Remark 2.2. Modules $M_{R}$ over a ring $R$ for which the mapping $M \otimes_{R} R \rightarrow$ $M$ given by $m \otimes r \mapsto m r$ is bijective are often called firm (see [31] for the first appearance of the term; earlier, J. L. Taylor [38] had called these modules regular, after which several authors used the name Taylor regular for them; since the term 'regular' has too many meanings even in ring theory, calling these modules 'firm' seems to be more practical). Firm acts have been used to develop Morita theory 
of semigroups in [27] and subsequent papers under the name 'closed acts'. Because of the obvious parallel between the module and the act case we prefer using the term 'firm act'. Note that this is also nicely consistent with the notation FAct S $_{S}$ (where, originally, the letter F stood for 'fixed', defined by Talwar [35] in a more complicated way).

It turns out that there are many firm semigroups. From the surjectivity of $\mu_{S}$ it follows that firm semigroups have to be factorisable. On the other hand, not every factorisable semigroup is firm.

Example 2.3. Consider the semigroup defined by the multiplication table

\begin{tabular}{l|llll} 
& 0 & $a$ & $b$ & $c$ \\
\hline 0 & 0 & 0 & 0 & 0 \\
$a$ & 0 & 0 & 0 & 0 \\
$b$ & 0 & 0 & 0 & $b$ \\
$c$ & 0 & $a$ & 0 & $c$
\end{tabular}.

Clearly, this semigroup is factorisable. It is easy to see, however, that the equivalence class $b \otimes a$ consists of only one pair, $(b, a)$, but there are other elements in the tensor product that map to 0 under $\mu$, hence the semigroup cannot be firm.

Proposition 2.4. A semigroup $S$ is firm in any of the following three cases.

(1) S has weak local units.

(2) $S$ has common weak right local units.

(3) $S$ has common weak left local units.

Proof. (1) This has been shown implicitly in [9, Lemma 4].

(2) Suppose that $S$ has common weak right local units. Then $S$ is factorisable and hence $\mu_{S}: S \otimes S \rightarrow S$ is surjective. Let now $s t=s^{\prime} t^{\prime}$, where $s, t, s^{\prime}, t^{\prime} \in S$. By assumption, there exists $v \in S$ such that $t v=t$ and $t^{\prime} v=t^{\prime}$. Hence we can calculate

$$
s \otimes t=s \otimes t v=s t \otimes v=s^{\prime} t^{\prime} \otimes v=s^{\prime} \otimes t^{\prime} v=s^{\prime} \otimes t^{\prime}
$$

in $S \otimes S$, which means that $\mu_{S}$ is also injective.

(3) is dual to (2).

Note that the conditions that appear in Proposition 2.4 are independent of each other.

Example 2.5. Let $S=\{0, a, e\}$ have the multiplication table

\begin{tabular}{l|lll} 
& 0 & $a$ & $e$ \\
\hline 0 & 0 & 0 & 0 \\
$a$ & 0 & 0 & $a$ \\
$e$ & 0 & 0 & $e$
\end{tabular}.

Here $e$ is a right identity of the semigroup $S$, so $S$ has common weak right local units. On the other hand, $S$ is neither a semigroup with weak local units nor a semigroup with common weak left local units.

Let now $T=\{e, f, 0\}$ be a semilattice with $e f=0$. Then $T$ has local units, but the elements $e$ and $f$ do not have a common weak right local unit.

Remark 2.6. The class of firm semigroups is rather big. Our computer calculations show that up to isomorphism there are 14448 factorisable semigroups of order 6 or less. Among those, 13344 are firm, while only 6853 have local units. 
Let us also point out the following relationship between firm semigroups and some other classes of semigroups.

Proposition 2.7. For a semigroup $S$ the following assertions are equivalent:

(1) $S$ is firm and fair,

(2) $S$ is factorisable and fair,

(3) S has weak local units.

Proof. The equivalence of (2) and (3) was shown in [25]. The implication (1) $\Rightarrow$ $(2)$ is obvious. The implication $(3) \Rightarrow(1)$ is valid by Proposition 2.4.

A right $S$-act $A_{S}$ is called nonsingular if $a=a^{\prime}\left(a, a^{\prime} \in A\right)$ whenever $a s=a^{\prime} s$ for all $s \in S$. Denote the category of unitary nonsingular right $S$-acts by $\mathrm{NAct}_{S}$. This category is used for developing Morita theory in [9]. (Notice that Chen and Shum [9] use the notation $U S$-FAct for our NAct ${ }_{S}$.) We point out that many results in Section 6 of [9] are obtained under the assumption that $\coprod_{i \in I} S \in S$-FxAct for any index set $I$ (see [9] for the definition of $S$-FxAct). This assumption is fulfilled for firm semigroups. Actually, this is almost proved in Lemma 4 of [9].

The act $S_{S}$ is nonsingular if and only if, for every $s, t \in S, s z=t z$ for all $z \in S$ implies $s=t$. Semigroups with this property are called right reductive.

The following two examples show that for a semigroup $S$, the categories FAct $_{S}$ and $\mathrm{NAct}_{S}$ are in general incomparable.

Example 2.8. Consider again the semigroup $S=\{0, a, e\}$ from Example 2.5. By Proposition 2.4, $S$ is a firm semigroup, and hence both $S_{S}$ and ${ }_{S} S$ are firm $S$-acts. Nevertheless, the left $S$-act ${ }_{S} S$ is not nonsingular, because $s 0=s a$ for each $s \in S$, but $0 \neq a$. This means that there exist firm left acts which are not nonsingular. Considering the dual of $S$ we can say a similar thing for right $S$-acts.

Example 2.9. Consider the semigroup $S$ defined by the multiplication table

\begin{tabular}{l|lllll} 
& 0 & $a$ & $b$ & $c$ & $d$ \\
\hline 0 & 0 & 0 & 0 & 0 & 0 \\
$a$ & 0 & 0 & 0 & 0 & $a$ \\
$b$ & 0 & 0 & 0 & 0 & $b$ \\
$c$ & 0 & 0 & $a$ & 0 & 0 \\
$d$ & 0 & $a$ & 0 & $c$ & $d$
\end{tabular}.

From this table it is easily seen that this semigroup is factorisable and right reductive, so the right act $S_{S}$ is unitary and nonsingular. Now $b c=00$, but $b \otimes c \neq 0 \otimes 0$ in $S \otimes S$, hence the act $S_{S}$ is not firm.

What we know is that $\mathrm{FAct}_{S}=\mathrm{NAct}_{S}$ if $S$ is a semigroup with common weak local units (this follows from Proposition 4 and Lemma 4 of [25]). However, even for semigroups with local units, these categories need not coincide.

Example 2.10. Let $S$ be a right zero semigroup with two or more elements. Then $S_{S}$ is firm, but it does not belong to $\mathrm{NAct}_{S}$. On the other hand, the one-element right $S$-act belongs to $\mathrm{NAct}_{S}$ but it is not firm.

In Proposition 3.13 we will see that these categories of acts are equivalent if $S$ is a firm semigroup.

Finally, we give a small table which compares some notations used in different sources. 


\begin{tabular}{c|c|c} 
this paper & Chen and Shum & $\begin{array}{c}\text { module case } \\
\text { (García and Marín) }\end{array}$ \\
\hline UAct $_{S}$ & $U S$-Act [9] & - \\
FAct $_{S}$ & - & DMod- $R[16]$ \\
NAct $_{S}$ & $U S$-FAct [9] & Mod- $R[16]$ \\
CAct $_{S}$ & - & CMod- $R[16]$
\end{tabular}

\section{Some AdJunctions Between ACT CATEgories}

In this section we study adjunctions between certain categories of acts. These results show that the different settings in which Morita equivalence of semigroups is considered in earlier papers amount to the same equivalence for firm semigroups. Some results obtained here for the category of firm acts will play a key role in later sections of the paper. The presentation of many results in this section is made uniform by using the notion of idempotent (co)pointed endofunctor.

If ${ }_{S} P_{T} \in{ }_{S} \operatorname{Act}_{T}$ and $B_{T} \in \operatorname{Act}_{T}$ then the hom-set $\operatorname{Act}_{T}(P, B)$ can be equipped with the canonical right $S$-action

$$
(f \cdot s)(p):=f(s \cdot p),
$$

$f \in \operatorname{Act}_{T}(P, B), s \in S, p \in P$. This allows us to consider functors

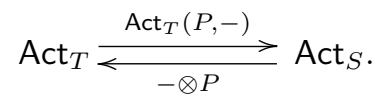

We have the usual adjunction between these two functors, with the expected unit and counit, given in the next lemma.

Lemma 3.1. Let $S$ and $T$ be semigroups, $A_{S} \in \operatorname{Act}_{S}, B_{T} \in \operatorname{Act}_{T}$ and ${ }_{S} P_{T} \in$ ${ }_{S} \mathrm{Act}_{T}$. The mappings

$$
\eta_{A}: A \longrightarrow \operatorname{Act}_{T}(P, A \otimes P), \quad a \mapsto(p \mapsto a \otimes p)
$$

and

$$
\varepsilon_{B}: \operatorname{Act}_{T}(P, B) \otimes P \longrightarrow B, \quad f \otimes p \mapsto f(p)
$$

are homomorphisms of right $S$-acts and T-acts, respectively, natural in $A$ and $B$.

Proof. To check that $\eta_{A}$ is a homomorphism of right $S$-acts, we calculate

$$
\eta_{A}(a s)(p)=a s \otimes p=a \otimes s p=\eta_{A}(a)(s p)=\left(\eta_{A}(a) \cdot s\right)(p) .
$$

Let $f: A_{S} \longrightarrow A_{S}^{\prime}$ be a morphism of right $S$-acts. We see that $\eta_{A}$ is natural in $A$ by calculating

$$
\begin{aligned}
\left(\operatorname{Act}_{T}(P, f \otimes P) \eta_{A}\right)(a)(p) & =\left(f \otimes 1_{P}\right)\left(\eta_{A}(a)(p)\right)=\left(f \otimes 1_{P}\right)(a \otimes p) \\
& =f(a) \otimes p=\eta_{A^{\prime}}(f(a))(p)=\left(\eta_{A^{\prime}} f\right)(a)(p) .
\end{aligned}
$$

To check that $\varepsilon_{B}$ is a homomorphism of right $T$-acts, we calculate

$$
\varepsilon_{B}((f \otimes p) t)=\varepsilon_{B}(f \otimes p t)=f(p t)=f(p) t=\varepsilon_{B}(f \otimes p) t .
$$


The naturality of $\varepsilon_{B}$ for a morphism $g: B_{T} \longrightarrow B_{T}^{\prime}$ of right $T$-acts is checked by calculating

$$
\begin{aligned}
\left(g \varepsilon_{B}\right)(f \otimes p) & =g(f(p))=\varepsilon_{B^{\prime}}(g f \otimes p)=\varepsilon_{B^{\prime}}\left(\left(\operatorname{Act}_{T}(P, g) \otimes 1_{P}\right)(f \otimes p)\right) \\
& =\left(\varepsilon_{B^{\prime}}\left(\operatorname{Act}_{T}(P, g) \otimes P\right)\right)(f \otimes p) .
\end{aligned}
$$

Proposition 3.2. Let $S$ and $T$ be semigroups and let ${ }_{S} P_{T} \in{ }_{S} \operatorname{Act}_{T}$. Then the functor $-\otimes P: \operatorname{Act}_{S} \longrightarrow \operatorname{Act}_{T}$ is left adjoint to the functor $\operatorname{Act}_{T}(P,-): \operatorname{Act}_{T} \rightarrow \operatorname{Act}_{S}$.

Proof. It is easy to check that the homomorphisms $\eta_{A}$ and $\varepsilon_{B}$, defined in Lemma 3.1, satisfy the triangle identities of the unit and counit of the adjunction in question.

Lemma 3.3. Let $S$ and $T$ be semigroups. Let $A_{S} \in \operatorname{Act}_{S}$ and ${ }_{S} P_{T} \in{ }_{S} \mathrm{Act}_{T}$. If $P$ is firm as a right $T$-act then $A \otimes P$ is also firm as a right $T$-act.

Proof. Let $P_{T}$ be firm. Clearly, the diagram

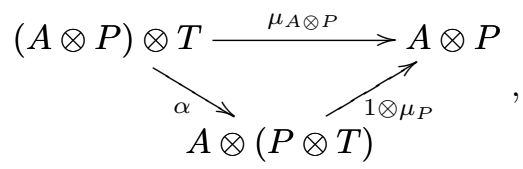

where $\alpha:(a \otimes p) \otimes t \mapsto a \otimes(p \otimes t)$, commutes. The statement follows because $\alpha$ and $1 \otimes \mu_{P}$ are isomorphisms.

Corollary 3.4. Let $S$ be a firm semigroup. Then $A \otimes S$ is a firm right $S$-act for any right $S$-act $A_{S}$ and $-\otimes S$ is a functor $\operatorname{Act}_{S} \rightarrow \mathrm{FAct}_{S}$.

It is easy to check that $\mu:-\otimes S \rightarrow 1_{\text {Act }_{S}}$ is a natural transformation.

Corollary 3.5. Let $S$ be a firm semigroup. Then we have

$$
\mu_{A} \otimes 1_{S}=\mu_{A \otimes S}:(A \otimes S) \otimes S \rightarrow A \otimes S,
$$

and this yields a natural isomorphism $(-\otimes S) \otimes S \longrightarrow-\otimes S:$ Act $_{S} \longrightarrow \operatorname{Act}_{S}$.

Proof. Take $P={ }_{S} S_{S}$ in Lemma 3.3 and notice that $\mu_{A} \otimes 1_{S}=\mu_{A \otimes S}$.

The last result tells us that the functor $-\otimes S$ is idempotent in some sense when $S$ is firm. For a firm semigroup $S$ the natural isomorphism $\mu$ makes the functor $-\otimes S$ into an idempotent copointed functor. Let us recall the definition of this notion, which enables us to prove several related results in a uniform way.

Definition 3.6. An endofunctor $F$ on a category $\mathcal{C}$ along with a natural transformation $\xi: 1_{\mathcal{C}} \longrightarrow F$ such that $\xi_{F(A)}=F\left(\xi_{A}\right): F(A) \longrightarrow F(F(A))$ is invertible for every $A \in \mathcal{C}$ is called an idempotent pointed endofunctor on $\mathcal{C}$. The dual notion (involving a natural transformation $\zeta: F \rightarrow 1_{\mathcal{C}}$ ) is called an idempotent copointed endofunctor.

The proof of the next lemma about (co)reflective subcategories can be found in Section 5.1 of [11]. First recall that a full replete subcategory $\mathcal{A}$ of a category $\mathcal{B}$ is said to be coreflective if the canonical inclusion functor admits a right adjoint. By the dual of Definition 3.5.6 in [7], a coreflective subcategory $\mathcal{A}$ of a category $\mathcal{B}$ is called an essential colocalization of $\mathcal{B}$ if the coreflection $\mathcal{B} \rightarrow \mathcal{A}$ admits a right adjoint. If $\kappa$ is a natural transformation between functors with domain $\mathcal{C}$, we 
say that an object $A$ of $\mathcal{C}$ is fixed by $\kappa$ when $\kappa_{A}$ is invertible. Let $\operatorname{Fix}(\mathcal{C}, \kappa)$ denote the full subcategory of $\mathcal{C}$ induced by the objects fixed by $\kappa$. Clearly, it is a replete subcategory of $\mathcal{C}$.

Lemma $3.7([11])$. If $(F, \xi)$ is an idempotent (co)pointed endofunctor on $\mathcal{C}$, then $\operatorname{Fix}(\mathcal{C}, \xi)$ is a (co)reflective subcategory of $\mathcal{C}$ with (co)reflection given by the corestriction of $F$ to $\operatorname{Fix}(\mathcal{C}, \xi)$. The adjunction unit in the copointed case is given by $\xi_{A}^{-1}: A \longrightarrow F(A)$.

The abovementioned corestriction will be denoted by $\left.F\right|^{\operatorname{Fix}(\mathcal{C}, \xi)}$. If the functor $F: \mathcal{C} \longrightarrow \mathcal{C}$ is a part of an adjunction, we can say more:

Lemma 3.8. Let $(F, \xi)$ be an idempotent copointed endofunctor on a category $\mathcal{C}$ and let $G: \mathcal{C} \longrightarrow \mathcal{C}$ be right adjoint to $F$ with adjunction unit $\eta$ and counit $\varepsilon$. Then

(1) the natural transformation $\zeta: 1 \longrightarrow G$ corresponding to $\xi: F \longrightarrow 1$ under the adjunction, defined componentwise as $\zeta_{A}=G\left(\xi_{A}\right) \eta_{A}$, makes $G$ into an idempotent pointed endofunctor on $\mathcal{C}$;

(2) we have

$$
\operatorname{Fix}(\mathcal{C}, \xi)=\operatorname{Fix}(\mathcal{C}, \varepsilon)
$$

and it is an essential colocalization of $\mathcal{C}$ with coreflection $\left.F\right|^{\mathrm{Fix}(\mathcal{C}, \xi)}$;

(3) we have

$$
\operatorname{Fix}(\mathcal{C}, \zeta)=\operatorname{Fix}(\mathcal{C}, \eta)
$$

and it is an essential localization of $\mathcal{C}$ with reflection $\left.G\right|^{\operatorname{Fix}(\mathcal{C}, \zeta)}$;

(4) the adjunction between $F$ and $G$ restricts to an adjoint equivalence

$$
\operatorname{Fix}(\mathcal{C}, \xi) \underset{G}{\stackrel{F}{\rightleftarrows}} \operatorname{Fix}(\mathcal{C}, \zeta) .
$$

Proof. (1) Recall (beginning of Section 1 of [10]) that if $L \dashv R$ and $L^{\prime} \dashv R^{\prime}$ are two adjunctions on a category $\mathcal{D}$, then there is a bijection between natural transformations $u: L \longrightarrow L^{\prime}$ and natural transformations $v: R^{\prime} \rightarrow R$ compatible with the vertical and horizontal composition of natural transformations. Since $\zeta$ corresponds to $\xi$ and $1_{F}$ to $1_{G}$, by compatibility $F \xi=\xi F$ being isomorphisms implies $\zeta G=G \zeta$ being isomorphisms.

(2) By Lemma 3.7 we have a coreflective subcategory $\operatorname{Fix}(\mathcal{C}, \xi)$ with coreflection functor $\left.F\right|^{\operatorname{Fix}(\mathcal{C}, \xi)}$. Clearly $\left.G\right|_{\operatorname{Fix}(\mathcal{C}, \xi)}$ is right adjoint to the coreflection, making $\operatorname{Fix}(\mathcal{C}, \xi)$ an essential colocalization of $\mathcal{C}$, which means that $\left.G\right|_{\operatorname{Fix}(\mathcal{C}, \xi)}$ is full and faithful according to Proposition 3.4.2 of [7]. Therefore $\varepsilon_{A}$ is invertible for objects $A$ from $\operatorname{Fix}(\mathcal{C}, \xi)$ (Proposition 3.4.1 of $[7]$ ). This gives us $\operatorname{Fix}(\mathcal{C}, \xi) \subseteq \operatorname{Fix}(\mathcal{C}, \varepsilon)$. To get the reverse inclusion, consider $A \in \operatorname{Fix}(\mathcal{C}, \varepsilon)$ and calculate

$$
\xi_{A} G\left(\varepsilon_{A}\right)=\varepsilon_{A} \xi_{F(G(A))}
$$

using the naturality of $\xi$. Since the morphisms other than $\xi_{A}$ involved here are invertible, $\xi_{A}$ must also be invertible, hence $A \in \operatorname{Fix}(\mathcal{C}, \xi)$.

(3) Analogous to (2).

(4) Note that any adjunction restricts to an adjoint equivalence between $\operatorname{Fix}(\mathcal{C}, \eta)$ and $\operatorname{Fix}(\mathcal{C}, \varepsilon)$ due to the triangle identities (Section 0.4 of [26]).

Note that in applying this result to the functor $-\otimes S: \operatorname{Act}_{S} \rightarrow \operatorname{Act}_{S}$, which has $\operatorname{Act}_{S}(S,-): \operatorname{Act}_{S} \longrightarrow \operatorname{Act}_{S}$ as a right adjoint (see Proposition 3.2), we want to 
know what is the idempotent pointed endofunctor corresponding to the idempotent copointed endofunctor $(-\otimes S, \mu)$. Let us denote it by $\left.\operatorname{Act}_{S}(S,-), \lambda\right)$. Then the natural map $\lambda_{A}: A \longrightarrow \operatorname{Act}_{S}(S, A)$ corresponds to $\mu_{A}: A \otimes S \longrightarrow A$ under the adjunction $-\otimes S \dashv \operatorname{Act}_{S}(S,-)$. We can calculate the action of $\lambda_{A}$ to be

$$
\lambda_{A}(a)=\operatorname{Act}_{S}\left(S, \mu_{A}\right)\left(\eta_{A}(a)\right)=\operatorname{Act}_{S}\left(S, \mu_{A}\right)(s \mapsto a \otimes s)=(s \mapsto a s) .
$$

Thus

$$
\lambda_{A}(a)(s)=a s
$$

for each $a \in A$ and $s \in S$. We will sometimes write $\lambda_{a}$ instead of $\lambda_{A}(a)$ for $a \in A$.

In the case of modules, a category denoted by CMod- $R$ (see, for example, [16]) has been used in investigations of Morita equivalence mainly in the works of Marín. Its act counterpart would be the full subcategory of Act $_{S}$ given by the acts $A_{S}$ for which $\lambda_{A}$ is invertible. We will denote this category by $\mathrm{CAct}_{S}$. Thus we have $\mathrm{CAct}_{S}=$ $\operatorname{Fix}\left(\operatorname{Act}_{S}, \lambda\right)$ and $\mathrm{FAct}_{S}=\operatorname{Fix}\left(\operatorname{Act}_{S}, \mu\right)$. The following proposition summarises the result of applying Lemma 3.8 to $-\otimes S: \operatorname{Act}_{S} \longrightarrow \operatorname{Act}_{S}$.

Proposition 3.9. Let $S$ be a firm semigroup. Then

(1) $\mathrm{FAct}_{S}$ is an essential colocalization of $\operatorname{Act}_{S}$ with coreflection $-\otimes S$;

(2) $\mathrm{CAct}_{S}$ is an essential localization of $\operatorname{Act}_{S}$ with reflection $\operatorname{Act}_{S}(S,-)$;

(3) there is an equivalence of categories

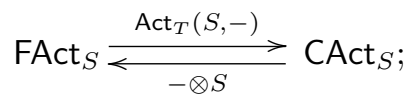

(4) a right $S$-act $A_{S}$ is firm if and only if $\varepsilon_{A}$ is invertible;

(5) a right $S$-act $A_{S}$ belongs to $\mathrm{CAct}_{S}$ if and only if $\eta_{A}$ is invertible.

Next we turn our attention to adjunctions related to $\mathrm{UAct}_{S}$. If $S$ is a factorisable semigroup and $A_{S}$ is a right $S$-act, then $A S=\{a s \mid a \in A, s \in S\}$ is the largest unitary subact of $A_{S}$. This construction is functorial as follows. If $f: A \longrightarrow B$ is a morphism of right $S$-acts, then $f S$ is the restriction of $f$ to $A S$ and corestriction to $B S$. The corestriction exists, since the image of a unitary act being unitary means that $f S$ must map $A S$ into $B S$.

We have the inclusion map $m_{A}: A S \rightarrow A$, which is obviously natural in $A$ and is easily seen to equip the functor $-S$ with an idempotent copointed endofunctor structure. Clearly, a right $S$-act $A_{S}$ is in $\mathrm{UAct}_{S}$ if and only if $m_{A}$ is invertible. Therefore by Lemma 3.7 we have:

Proposition 3.10. Let $S$ be a factorisable semigroup. Then the inclusion functor $I:$ UAct $_{S} \longrightarrow$ Act $_{S}$ is left adjoint to the functor $-S:$ Act $_{S} \rightarrow$ UAct $_{S}$, with adjunction unit given by $m_{A}^{-1}: A \longrightarrow A S$.

The functor $-S$ does not in general have a right adjoint, even when $S$ is firm.

Example 3.11. Let $S$ be a non-singleton right zero semigroup. Let $A_{S}=S_{S}$ and let $\nabla=A \times A$ be the largest congruence on $A_{S}$. The congruence $\nabla$ is also an $S$-act, and $\nabla S$ is the equality relation $\Delta$. Obviously, $A / \nabla=\{*\}$ and $(A S) /(\nabla S)=$ $A / \Delta=A$ are nonisomorphic.

Colimits in Act $_{S}$ are calculated on the level of sets exactly as in Set and the structure maps on colimits are induced in the natural way. Therefore what we have shown is that the functor $-S:$ Act $_{S} \longrightarrow$ Act $_{S}$ does not preserve the coequalizer of 
the two projections $\nabla \rightarrow A$ of the congruence $\nabla$ and therefore it cannot have a right adjoint.

If $S$ is firm, then the functor $-\otimes S: \mathrm{UAct}_{S} \longrightarrow \mathrm{UAct}_{S}$ does have a right adjoint, which turns out to be $\operatorname{Act}_{S}(S,-) S: \mathrm{UAct}_{S} \longrightarrow \mathrm{UAct}_{S}$. To see that, compose the adjunctions $-\otimes S \dashv \operatorname{Act}_{S}(S,-): \operatorname{Act}_{S} \longrightarrow \operatorname{Act}_{S}$ and $I \dashv-S: \operatorname{Act}_{S} \longrightarrow$ UAct $_{S}$ to get the adjunction

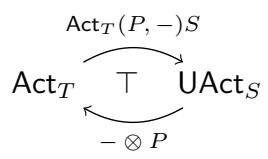

and observe that we can restrict the left category to $\mathrm{UAct}_{S}$, since $-\otimes S$ is unitary for firm $S$. Denote the unit and counit of this adjunction by $\eta^{\prime}$ and $\varepsilon^{\prime}$. We can use the adjunction $I \dashv-S:$ Act $_{S} \longrightarrow$ UAct $_{S}$ with unit $m^{-1}: 1 \longrightarrow-S$ and counit $m_{A}: A S \longrightarrow A$ to calculate

$$
\eta_{A}^{\prime}=\left(\eta_{A} S\right) m_{A}^{-1} \quad \text { and } \quad \varepsilon_{A}^{\prime}=\varepsilon_{A}\left(m_{\text {Act }_{S}(S, A)} \otimes 1_{S}\right) .
$$

Now $\mu:-\otimes S \longrightarrow 1$ makes $-\otimes S$ into an idempotent copointed endofunctor on $\mathrm{UAct}_{S}$ posessing a right adjoint. Applying Lemma 3.8 to it, we once again ask what is the idempotent pointed endofunctor on $\operatorname{Act}(S,-) S$ corresponding under the adjunction to $\mu_{A}: A \otimes S \longrightarrow A$. Denote the map $A \longrightarrow \operatorname{Act}_{S}(S, A) S$ by $\lambda_{A}^{\prime}$ and compute

$$
\begin{aligned}
\lambda_{A}^{\prime} & =\operatorname{Act}_{S}\left(S, \mu_{A}\right) S \circ \eta_{A}^{\prime}=\operatorname{Act}_{S}\left(S, \mu_{A}\right) S \circ \eta_{A} S \circ m_{A}^{-1} \\
& =\left(\operatorname{Act}_{S}\left(S, \mu_{A}\right) \eta_{A}\right) S \circ m_{A}^{-1}=\left(\lambda_{A} S\right) m_{A}^{-1} .
\end{aligned}
$$

This shows that $\lambda^{\prime}$ is essentially the corestriction of $\lambda_{A}$ to $\operatorname{Act}_{S}(S, A) S$, which exists if $A_{S}$ is unitary. We now need to identify the essential localization of UAct ${ }_{S}$ that Lemma 3.8 gives us.

Proposition 3.12. Let $S$ be a semigroup and $A_{S} \in \mathrm{UAct}_{S}$. Then the mapping

$$
\lambda_{A}^{\prime}: A \longrightarrow \operatorname{Act}_{S}(S, A) S, \quad a \mapsto \lambda_{a}
$$

is surjective and $A_{S}$ is in $\mathrm{NAct}_{S}$ if and only if $\lambda_{A}^{\prime}$ is bijective.

Proof. Notice that $f \cdot s=\lambda_{f(s)}$, since

$$
(f \cdot s)(z)=f(s z)=f(s) z=\lambda_{f(s)}(z) .
$$

This implies that $\lambda_{A}^{\prime}$ is surjective for any unitary $A_{S}$. By definition, a unitary right $S$-act $A_{S}$ is in $\mathrm{NAct}_{S}$ if and only if the mapping $\lambda_{A}: A \rightarrow \operatorname{Act}_{S}(S, A)$ is injective. Clearly, $\lambda_{A}$ is injective if and only if $\lambda_{A}^{\prime}$ is injective. Thus the result follows.

We can now summarise the result of applying Lemma 3.8 to the idempotent copointed endofunctor $(-\otimes S, \mu)$ on $\mathrm{UAct}_{S}$.

Proposition 3.13. Let $S$ be firm semigroup. Then

(1) FAct $_{S}$ is an essential colocalization of $\mathrm{UAct}_{S}$ with coreflection $-\otimes S$;

(2) $\mathrm{NAct}_{S}$ is an essential localization of $\mathrm{UAct}_{S}$ with reflection $\operatorname{Act}_{S}(S,-) S$;

(3) there is an equivalence of categories

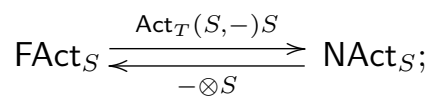


(4) a unitary right $S$-act $A_{S}$ is firm if and only if $\varepsilon_{A}^{\prime}$ is invertible;

(5) a unitary right $S$-act $A_{S}$ belongs to $\mathrm{NAct}_{S}$ if and only if $\eta_{A}^{\prime}$ is invertible.

Remark 3.14. Notice that for claim (2) it suffices to require only that $S$ is factorisable. Namely, in that case $A \otimes S$ is unitary for $A_{S} \in \mathrm{NAct}_{S}$, hence we may consider the functor $-\otimes S: \mathrm{NAct}_{S} \longrightarrow \mathrm{UAct}_{S}$, and this functor admits $\operatorname{Act}_{S}(S,-) S: \mathrm{UAct}_{S} \longrightarrow \mathrm{NAct}_{S}$ as its right adjoint.

There is a remarkably strong result in [28] (Proposition 2.7), which says that, for an idempotent ring (the counterpart of a factorisable semigroup in ring theory), the category of firm modules (DMod- $R$ ) is equivalent to the category Mod- $R$ whose objects are modules $M_{R}$ such that $M R=M$ and, for all $m \in M$, if $m R=0$ then $m=0$ (the counterpart of $\mathrm{NAct}_{S}$ ), and also to the category CMod- $R$. In the semigroup case we have as an immediate consequence of Propositions 3.9 and 3.12:

Theorem 3.15. For a firm semigroup $S$, the categories FAct $_{S}$, NAct $_{S}$ and CAct $_{S}$ are equivalent.

In contrast to the ring case, we are not able to prove that Theorem 3.15 holds for every factorisable semigroup $S$.

The following diagram summarizes adjunctions and equivalences between act categories for a firm semigroup $S$. Here $I$ stands for (different) inclusion functors.

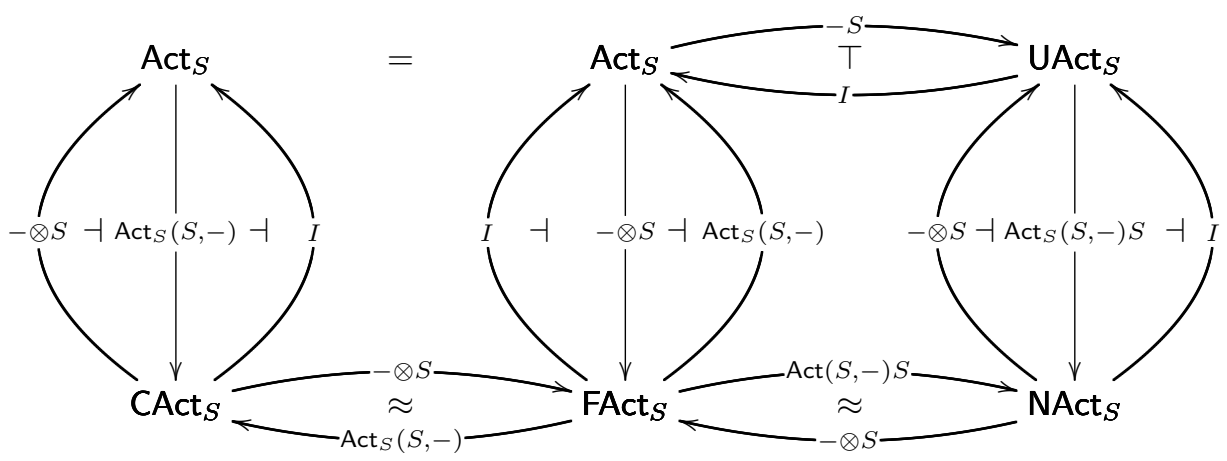

The equivalence between $\mathrm{CAct}_{S}$ and $\mathrm{NAct}_{S}$ can be established directly by the functors

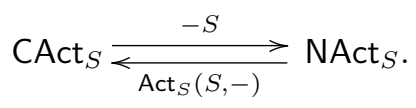

We can combine the information about firm acts to obtain the following result.

Theorem 3.16. Let $S$ be a firm semigroup and $A_{S}$ a unitary right $S$-act. Then the following assertions are equivalent.

(1) $A_{S}$ is firm.

(2) There exists an isomorphism $A \otimes S \rightarrow A$ of right $S$-acts.

(3) $\varepsilon_{A}$ is invertible.

(4) $\varepsilon_{A}^{\prime}$ is invertible. 
Proof. (1) $\Rightarrow(2)$. This is obvious.

$(2) \Rightarrow(1)$. Since $A \otimes S$ is firm, the existence of an isomorphism $A \otimes S \rightarrow A$ means that $A_{S}$ is also firm.

(1) $\Leftrightarrow(3)$ by Proposition 3.9.

(1) $\Leftrightarrow$ (4) by Proposition 3.13.

In [27, Proposition 2.3], Lawson showed that, for a semigroup $S$ with local units, the category ${ }_{S}$ FAct coincides with the category consisting of left $S$-acts ${ }_{S} A$ for which the canonical mapping $S \otimes S \operatorname{Act}(S, A) \rightarrow A$ is bijective. Acts fixed by $\varepsilon$ over semigroups with local units were introduced by Talwar in [35]. In a subsequent paper [36], he used acts fixed by

$$
\Gamma_{A}: \operatorname{Act}_{S}(S, A) S \otimes S \rightarrow A, \quad f \otimes s \mapsto f(s)
$$

(written in left-right dual) to develop Morita theory for factorisable semigroups. The homomorphism $\Gamma$ is the same as $\varepsilon^{\prime}$ from Proposition 3.13 because

$$
\varepsilon_{A}^{\prime}(f \otimes s)=\left(\varepsilon_{A}\left(m_{\operatorname{Act}_{S}(S, A)} \otimes 1_{S}\right)\right)(f \otimes s)=\varepsilon_{A}(f \otimes s)=f(s) .
$$

Theorem 3.16 yields immediately the following generalisation of Lawson's result to firm semigroups.

Corollary 3.17. Over a firm semigroup, firm acts are the same as fixed acts in the sense of Talwar [35] and [36].

Theorem 3.16 has also another interesting corollary. For this, observe that a semigroup operation can be defined on $\operatorname{Act}_{S}(S, S) \otimes S$ by putting

$$
(f \otimes s)\left(f^{\prime} \otimes s^{\prime}\right):=f \otimes s f^{\prime}\left(s^{\prime}\right) .
$$

It is straightforward to check that this multiplication is associative.

Corollary 3.18. If $S$ is a firm semigroup then

$$
S \cong \operatorname{Act}_{S}(S, S) \otimes S
$$

both as $(S, S)$-biacts and as semigroups.

Proof. By Theorem 3.16, the mapping $\varepsilon_{S}: \operatorname{Act}_{S}(S, S) \otimes S \rightarrow S, f \otimes s \mapsto f(s)$ (see Lemma 3.1) is an isomorphism of right $S$-acts. The left $S$-action on $\operatorname{Act}_{S}(S, S)$ is defined by

$$
(s \cdot f)(z):=s f(z)
$$

$s, z \in S, f \in \operatorname{Act}_{S}(S, S)$. Now $\varepsilon_{S}$ is a homomorphism of left $S$-acts because

$$
\varepsilon_{S}(z(f \otimes s))=\varepsilon_{S}((z \cdot f) \otimes s)=(z \cdot f)(s)=z f(s)=z \varepsilon_{S}(f \otimes s)
$$

for every $z, s \in S$ and $f \in \operatorname{Act}_{S}(S, S)$. This proves that $\varepsilon_{S}$ is an $(S, S)$-biact isomorphism. It is also a semigroup homomorphism because

$$
\begin{aligned}
\varepsilon_{S}\left((f \otimes s)\left(f^{\prime} \otimes s^{\prime}\right)\right) & =\varepsilon_{S}\left(f \otimes s f^{\prime}\left(s^{\prime}\right)\right)=f\left(s f^{\prime}\left(s^{\prime}\right)\right)=f(s) f^{\prime}\left(s^{\prime}\right) \\
& =\varepsilon_{S}(f \otimes s) \varepsilon_{S}\left(f^{\prime} \otimes s^{\prime}\right) .
\end{aligned}
$$

As in [9], for a right $S$-act $A_{S}$ we consider the congruence

$$
\zeta_{A}=\left\{\left(a_{1}, a_{2}\right) \in A^{2} \mid a_{1} s=a_{2} s \text { for all } s \in S\right\} .
$$

Since $\zeta_{A}$ is the kernel of $\lambda_{A}$, and of $\lambda_{A}^{\prime}$ if $A_{S}$ is unitary, we have the following: 
Proposition 3.19. Let $S$ be a semigroup and $A_{S}$ be a unitary right $S$-act. Then the right $S$-acts $\operatorname{Act}(S, A) S$ and $A / \zeta_{A}$ are isomorphic.

Proof. From the proof of Proposition 3.12 we see that the map $\lambda_{A}^{\prime}: A \rightarrow \operatorname{Act}_{S}(S, A) S$ is surjective. Since $\zeta_{A}=\operatorname{ker}\left(\lambda_{A}^{\prime}\right)$, the Homomorphism Theorem yields an isomorphism as required.

Corollary 3.20. If $S$ is a firm semigroup then

(1) $S / \zeta_{S}$ is a generator in $\mathrm{NAct}_{S}$;

(2) $S_{S}$ is a generator in FAct $_{S}$;

(3) $\operatorname{Act}_{S}(S, S)$ is a generator in $\mathrm{CAct}_{S}$.

Proof. (1) This is Lemma 2(iv) in [9].

(2) Applying the equivalence functor $-\otimes S: \mathrm{NAct}_{S} \rightarrow \mathrm{FAct}_{S}$ to the generator $S / \zeta_{S}$ we obtain a generator $S / \zeta_{S} \otimes S$ in FAct $_{S}$. By Proposition 3.19, this is isomorphic to $\operatorname{Act}(S, S) S \otimes S$, which by part (4) of Theorem 3.16 is isomorphic to $S_{S}$. Therefore $S_{S}$ is also a generator in FAct ${ }_{S}$.

(3) We apply the equivalence functor $\operatorname{Act}_{S}(S,-): \mathrm{FAct}_{S} \rightarrow \mathrm{CAct}_{S}$ to the generator $S_{S}$.

\section{Equivalence FUnCTORS BetweEn CATEGories OF FIRM ACTS}

The goal of this section is to prove an analogue of the Eilenberg-Watts theorem, stating that equivalence functors between categories of firm acts over firm semigroups are naturally isomorphic to tensor multiplication functors. The proof will be based on Theorem 3.16.

We begin with the following

Proposition 4.1. Let $S$ and $T$ be firm semigroups and ${ }_{S} P_{T}$ be a biact such that $P_{T}$ is firm. Then the functor $-\otimes P:$ FAct $_{S} \longrightarrow$ FAct $_{T}$ is left adjoint to the functor $\operatorname{Act}_{T}(P,-) \otimes S:$ FAct $_{T} \longrightarrow$ FAct $_{S}$.

Proof. If we compose the adjunction in Proposition 3.2 with the adjunction between the inclusion and coreflection of the category FAct $_{S}$ (see Proposition 3.9), we get the adjunction

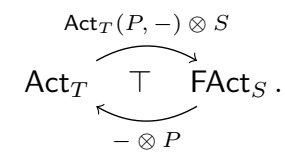

Since firmness of $P_{T}$ implies that the image of $-\otimes P$ lies in FAct $_{T}$ (see Lemma 3.3), we obtain the required result.

Composing this adjunction with the adjunction in Proposition 3.10 we get two right adjoints $-\otimes S$ and $-S \otimes S$ to the inclusion functor FAct $\rightarrow$ Act $_{S}$, which means that the right adjoints must be isomorphic.

Whenever $F:$ FAct $_{S} \rightarrow$ FAct $_{T}$ and $G:$ FAct $_{S} \rightarrow$ FAct $_{T}$ are mutually inverse equivalence functors, we can turn $P:=F(S)_{T}$ and $Q:=G(T)_{S}$ into a left $S$-act and a left $T$-act, respectively, by putting

$$
\begin{aligned}
s \cdot p & :=F\left(l_{s}\right)(p), \\
t \cdot q & :=G\left(l_{t}\right)(q),
\end{aligned}
$$


where $p \in P, q \in Q$, and

$$
\begin{aligned}
& l_{s}: S_{S} \rightarrow S_{S}, u \mapsto s u, \\
& l_{t}: T_{T} \rightarrow T_{T}, v \mapsto t v .
\end{aligned}
$$

Therefore we will have biacts ${ }_{S} P_{T}$ and ${ }_{T} Q_{S}$.

Proposition 4.2. Suppose that $S$ and $T$ are firm semigroups and $F:$ FAct $_{S} \rightarrow$ FAct $_{T}$ and $G:$ FAct $_{S} \rightarrow$ FAct $_{T}$ are mutually inverse equivalence functors. Then

$$
F \cong \operatorname{Act}_{S}(G(T),-) \otimes T \quad \text { and } \quad G \cong \operatorname{Act}_{T}(F(S),-) \otimes S .
$$

Proof. Because $F$ and $G$ are equivalence functors, we have an isomorphism

$$
\omega_{A}: \operatorname{Act}_{T}(T, F(A)) \rightarrow \operatorname{Act}_{S}(G(T), A)
$$

in Set which is natural in $A_{S} \in \mathrm{FAct}_{S}$. We will show that actually this is also an isomorphism in $\operatorname{Act}_{T}$. Using (3.1) we see that $\operatorname{Act}_{S}(G(T), A)$ is a right $T$-act with the action

$$
f \cdot t=f \circ G\left(l_{t}\right)
$$

and $\operatorname{Act}_{T}(T, F(A))$ is a right $T$-act with the action

$$
h \cdot t=h \circ l_{t} .
$$

For every $t \in T$, the diagram

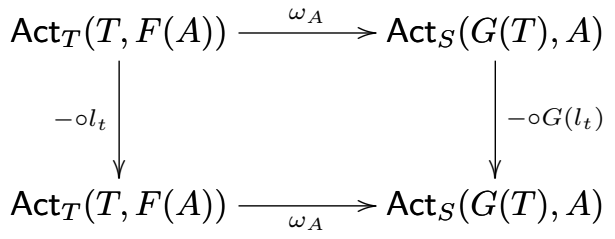

commutes because of the naturality of $\omega$. Thus

$$
\omega_{A}(h \cdot t)=\omega_{A}\left(h \circ l_{t}\right)=\omega_{A}(h) \circ G\left(l_{t}\right)=\omega_{A}(h) \cdot t,
$$

and $\omega_{A}$ is a right $T$-homomorphism.

Since $F(A)_{T}$ is firm, it is also fixed by Theorem 3.16, and hence

$$
F(A) \cong \operatorname{Act}_{T}(T, F(A)) \otimes T \cong \operatorname{Act}_{S}(G(T), A) \otimes T,
$$

where the isomorphisms are natural in $A_{S} \in \mathrm{FAct}_{S}$. Hence $F \cong \operatorname{Act}_{S}(G(T),-) \otimes T$. The other isomorphism can be proved similarly.

Theorem 4.3. Let $S$ and $T$ be firm semigroups and let $F:$ FAct $_{S} \longrightarrow$ FAct $_{T}$ and $G:$ FAct $_{S} \longrightarrow \mathrm{FAct}_{T}$ be mutually inverse equivalence functors. Then

$$
\begin{aligned}
& F \cong-\otimes F(S), \\
& G \cong-\otimes G(T) .
\end{aligned}
$$

Moreover, the left acts ${ }_{S} F(S)$ and ${ }_{T} G(T)$ are firm.

Proof. By Proposition 4.2, $G$ is naturally isomorphic to $\operatorname{Act}_{T}(F(S),-) \otimes S$. By Proposition 4.1, this functor has $-\otimes F(S)$ as its left adjoint. Since $F$, being the inverse equivalence functor of $G$, is also a left adjoint of $G$, we get that $F \cong-\otimes F(S)$. A similar argument for $G$ gives $G \cong-\otimes G(T)$. 
Let $\nu: F \rightarrow-\otimes F(S)$ be a natural isomorphism. Then $\nu_{S}: F(S) \rightarrow S \otimes F(S)$ is an isomorphism of right $T$-acts, therefore it is bijective. Take $s \in S$ and $p \in P$, and let $\nu_{S}(p)=s^{\prime} \otimes p^{\prime}$. Then, by naturality of $\nu$, the diagram

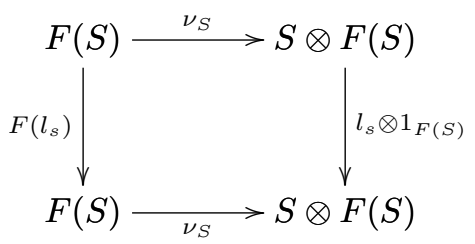

commutes and we can calculate:

$$
\begin{aligned}
\nu_{S}(s \cdot p) & =\nu_{S}\left(F\left(l_{s}\right)(p)\right)=\left(l_{s} \otimes 1_{F(S)}\right)\left(\nu_{S}(p)\right)=\left(l_{s} \otimes 1_{F(S)}\right)\left(s^{\prime} \otimes p^{\prime}\right) \\
& =s s^{\prime} \otimes p^{\prime}=s\left(s^{\prime} \otimes p^{\prime}\right)=s \nu_{S}(p) .
\end{aligned}
$$

This means that $\nu_{S}$ is a homomorphism of left $S$-acts. By the analogue of Theorem 3.16 for left acts, this implies that the left act ${ }_{S} F(S)$ is firm. For the same reason, the left act ${ }_{T} G(T)$ is firm.

\section{Morita equivalence and Strong Morita equivalence CoIncide For FIRM SEMIGROUPS}

In this section we will prove that right Morita equivalence and strong Morita equivalence coincide on the class of firm semigroups. Let us recall some definitions.

Definition 5.1 ([36]). A Morita context is a six-tuple $\left(S, T,{ }_{S} P_{T},{ }_{T} Q_{S}, \theta, \phi\right)$, where $S$ and $T$ are semigroups, ${ }_{S} P_{T} \in{ }_{S} \operatorname{Act}_{T}$ and ${ }_{T} Q_{S} \in{ }_{T} \mathrm{Act}_{S}$ are biacts, and

$$
\theta:{ }_{S}(P \otimes Q)_{S} \rightarrow{ }_{S} S_{S}, \quad \phi:{ }_{T}(Q \otimes P)_{T} \rightarrow{ }_{T} T_{T}
$$

are biact morphisms such that, for every $p, p^{\prime} \in P$ and $q, q^{\prime} \in Q$,

$$
\theta(p \otimes q) p^{\prime}=p \phi\left(q \otimes p^{\prime}\right), \quad q \theta\left(p \otimes q^{\prime}\right)=\phi(q \otimes p) q^{\prime} .
$$

We say that a Morita context $\left(S, T,{ }_{S} P_{T},{ }_{T} Q_{S}, \theta, \phi\right)$ is

- unitary, if ${ }_{S} P_{T}$ and ${ }_{T} Q_{S}$ are unitary biacts,

- surjective, if $\theta$ and $\phi$ are surjective,

- bijective, if $\theta$ and $\phi$ are bijective.

Definition 5.2 ([36]). Semigroups $S$ and $T$ are called strongly Morita equivalent if they are contained in a unitary surjective Morita context.

Definition 5.3. We say that semigroups $S$ and $T$ are right Morita equivalent if the categories $\mathrm{FAct}_{S}$ and $\mathrm{FAct}_{T}$ are equivalent.

First of all, let us mention that the relations of right Morita equivalence on the class of all semigroups and the relation of strong Morita equivalence on the class of factorisable semigroups are equivalence relations (factorisability is necessary for the reflexivity of strong Morita equivalence). One of the central questions in Morita theory is: when these two relations coincide. If they coincide, then instead of using functors and natural transformations one can use Morita contexts for studying various problems (for example studying Morita invariants).

While it is clear what is meant by strong Morita equivalence, it is not so obvious what right Morita equivalence should mean. In different articles, various categories have been used to define Morita equivalence. In the present text we have shown 
that, at least for firm semigroups, it does not make any difference which of these categories one uses. For us, the category of firm acts seems to be the most natural choice.

For semigroups with local units we know the following.

Theorem 5.4 ([27, Theorem 1.1]). Right Morita equivalence and strong Morita equivalence coincide on the class of semigroups with local units.

The proof relies heavily on the fact that the acts $e S$, where $e$ is an idempotent, are indecomposable projectives in $\mathrm{FAct}_{S}$. But an arbitrary semigroup may have no idempotents at all. So if one wants to extend this result to larger classes of semigroups, it is necessary to take a different approach. One possibility is to use generators instead of indecomposable projectives, as is done, for example, in [9]. In that article, Chen and Shum consider the category of unitary nonsingular acts in place of our category of firm acts. What they obtain is that equivalence of the categories of these acts is equivalent to the existence of a surjective Morita context which, instead of $S$ and $T$, involves quotients of $S$ and $T$ by the congruences $\zeta_{S}$ and $\zeta_{T}$.

Theorem 5.5 ([9, Theorem 3]). Let $S$ and $T$ be factorisable semigroups. The categories $\mathrm{NAct}_{S}$ and $\mathrm{NAct}_{T}$ are equivalent if and only if the semigroups $S / \zeta_{S}$ and $T / \zeta_{T}$ are strongly Morita equivalent.

Remark 5.6. In view of Proposition 3.13(3), this implies that two firm semigroups $S$ and $T$ are (strongly) Morita equivalent if and only if $S / \zeta_{S}$ and $T / \zeta_{S}$ are strongly Morita equivalent. Here the adjective 'strongly' cannot be omitted, in other words, we cannot write that $\mathrm{FAct}_{S}$ and $\mathrm{FAct}_{T}$ are equivalent if and only if $\mathrm{FAct}_{S / \zeta}$ and FAct $_{T / \zeta_{T}}$ are equivalent because it may happen that a semigroup $S$ is firm but $S / \zeta_{S}$ is not. Indeed, take

\begin{tabular}{|c|c|c|c|c|c|c|c|c|c|c|c|c|}
\hline & 0 & $a$ & $b$ & $c$ & $d$ & $e$ & & & $\overline{0}$ & & & \\
\hline 0 & 0 & 0 & 0 & 0 & 0 & 0 & & $\overline{0}$ & $\overline{\bar{\Omega}}$ & $\overline{0}$ & $\frac{c}{0}$ & $\overline{0}$ \\
\hline$a$ & 0 & 0 & 0 & 0 & 0 & 0 & & $\bar{b}$ & $\overline{0}$ & $\overline{0}$ & $\overline{0}$ & \\
\hline$S=b$ & 0 & 0 & 0 & 0 & 0 & $b$ & $S / \zeta_{S}=$ & $b$ & $\overline{0}$ & $\frac{0}{0}$ & $\frac{0}{0}$ & 0 \\
\hline$c$ & 0 & 0 & 0 & 0 & $a$ & $c$ & & $\frac{c}{d}$ & $\overline{0}$ & $\overline{0}$ & $\frac{U}{h}$ & $\frac{a}{0}$ \\
\hline$d$ & 0 & 0 & 0 & $b$ & 0 & 0 & & $\bar{a}$ & $\overline{0}$ & $\bar{b}$ & $\frac{0}{0}$ & \\
\hline$e$ & 0 & 0 & $b$ & 0 & $d$ & $e$ & & $e$ & & & & \\
\hline
\end{tabular}

where

$$
\zeta_{S}=\{(0,0),(a, a),(b, b),(c, c),(d, d),(e, e),(0, a),(a, 0)\}
$$

Another possible direction is to study fair semigroups, which need not even be factorisable. For fair semigroups one gets a Morita context in which $S$ and $T$ are replaced by certain ideals of $S$ and $T$. Put $U(S)=\{s \in S \mid s=u s=$ $s v$ for some $u, v \in S\}$, which is an ideal of $S$.

Theorem 5.7 ([25, Proposition 3.12]). Let $S$ and $T$ be fair semigroups such that $U(S)$ and $U(T)$ have common weak local units. Then $S$ and $T$ are right Morita equivalent if and only if $U(S)$ and $U(T)$ are strongly Morita equivalent.

The question arises: how far can we go from semigroups with local units without replacing $S$ and $T$ in the Morita context by something else? We will show in this section that we can go at least to the class of firm semigroups. 
One of the canonical examples of bicategories is rings with identity, bimodules, and bimodule homomorphisms (see [7], Example 7.7.4). In the same way, monoids, biacts and act homomorphisms form a bicategory, and so do firm semigroups, firm biacts and acts homomorphisms. The objects of the latter bicategory are firm semigroups, the 1-cells are firm biacts and are composed using the tensor product, the 2-cells are act homomorphisms. The fact that these data form a bicategory follows essentially in the same way as it does for rings. The only difference to note is that we do need the semigroups and biacts in this construction to be firm, since only then will our bicategory have the ${ }_{S} S_{S}$ as unit 1-cells, since the requirement of a right $S$-act being firm is essentially the same as saying that ${ }_{S} S_{S}$ is a right unit for the composition in that bicategory.

The following lemma is a standard 2-categorical fact, see, for example, Proposition 1.1 in [13]; we formulate it in our special case.

Lemma 5.8. Let $S$ and $T$ be firm semigroups, let ${ }_{S} P_{T}$ and ${ }_{T} Q_{S}$ be firm biacts and suppose that there are isomorphisms $\theta: P \otimes Q \rightarrow S$ in ${ }_{S}$ Act $_{S}$ and $\phi: Q \otimes P \rightarrow T$ in ${ }_{T} \mathrm{Act}_{T}$. Then there there exists an isomorphism $\phi^{\prime}: Q \otimes P \longrightarrow T$ in ${ }_{T} \mathrm{Act}_{T}$ such that $\left\langle S, T,{ }_{S} P_{T},{ }_{T} Q_{S}, \theta, \phi^{\prime}\right\rangle$ is a Morita context.

Now we are ready to prove our main result. It is the non-additive counterpart of a theorem announced by Quillen [32] in 1996 stating that any Morita equivalence between firm rings is given by a unique Morita context. Quillen presented the theorem in a lecture at the University of Exeter but has not published it. The result was rediscovered by García and Marín in 1999 and appeared as Proposition 18 in [16], without using the terms 'firm ring' and 'firm module'.

Theorem 5.9. Let $S$ and $T$ be firm semigroups. The following assertions are equivalent.

(1) The categories $\mathrm{FAct}_{S}$ and $\mathrm{FAct}_{T}$ are equivalent.

(2) The categories ${ }_{S} \mathrm{FAct}$ and ${ }_{T} \mathrm{FAct}$ are equivalent.

(3) There exists a unitary bijective Morita context containing $S$ and $T$.

(4) There exists a unitary surjective Morita context containing $S$ and $T$.

(5) There exists a surjective Morita context containing $S$ and $T$.

Proof. (1) $\Rightarrow$ (3). Let $F:$ FAct $_{S} \rightarrow$ FAct $_{T}$ and $G:$ FAct $_{T} \rightarrow$ FAct $_{S}$ be mutually inverse equivalence functors. As before, we may consider the biacts ${ }_{S} P_{T}=F(S)$ and ${ }_{T} Q_{S}=G(T)$. Since $P_{T}$ and $Q_{S}$ are firm, $P_{T}$ and $Q_{S}$ are unitary right acts. According to Theorem 4.3, ${ }_{S} P$ and ${ }_{T} Q$ are firm (and hence unitary) left acts. By the same result, $F \cong-\otimes P$ and $G \cong-\otimes Q$. Thus also

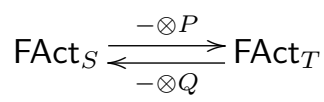

are mutually inverse equivalence functors. This means that there are isomorphisms

$$
\begin{aligned}
& \alpha_{A}:(A \otimes P) \otimes Q \rightarrow A \text { in } \text { Act }_{S}, \\
& \beta_{B}:(B \otimes Q) \otimes P \longrightarrow B \quad \text { in } \text { Act }_{T},
\end{aligned}
$$


natural in $A_{S} \in \mathrm{FAct}_{S}$ and $B_{T} \in \mathrm{FAct}_{T}$, respectively. Let us show that the mapping $\alpha_{S}:(S \otimes P) \otimes Q \rightarrow S$ is a homomorphism of left $S$-acts. Since the square

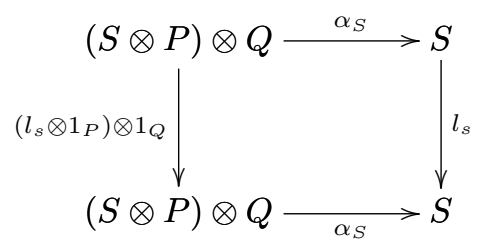

commutes, we have

$$
\begin{aligned}
s \alpha_{S}\left(\left(s^{\prime} \otimes p\right) \otimes q\right) & =\left(l_{s} \alpha_{S}\right)\left(\left(s^{\prime} \otimes p\right) \otimes q\right)=\left(\alpha_{S}\left(\left(l_{s} \otimes 1_{P}\right) \otimes 1_{Q}\right)\right)\left(\left(s^{\prime} \otimes p\right) \otimes q\right) \\
& =\alpha_{S}\left(\left(s s^{\prime} \otimes p\right) \otimes q\right)=\alpha_{S}\left(s\left(\left(s^{\prime} \otimes p\right) \otimes q\right)\right)
\end{aligned}
$$

for every $s, s^{\prime} \in S, p \in P$ and $q \in Q$. Thus $\alpha_{S}$ is an isomorphism in ${ }_{S} \operatorname{Act}_{S}$.

Since ${ }_{S} P$ is firm, the mapping

$$
\nu_{P}: S \otimes P \rightarrow P, \quad s \otimes p \mapsto s \cdot p
$$

is an isomorphism in ${ }_{S}$ Act. Clearly, it is also an isomorphism in ${ }_{S}$ Act $_{T}$. Applying the functor $-\otimes Q:{ }_{S} \mathrm{Act}_{T} \rightarrow{ }_{S} \mathrm{Act}_{S}$ to the isomorphism $\nu_{P}^{-1}: P \rightarrow S \otimes P$ in ${ }_{S} \mathrm{Act}_{T}$ gives an isomorphism $\nu_{P}^{-1} \otimes 1_{Q}: P \otimes Q \rightarrow(S \otimes P) \otimes Q$ in ${ }_{S}$ Act $_{S}$. Denoting the composite

$$
P \otimes Q \stackrel{\nu_{P}^{-1} \otimes 1_{Q}}{\longrightarrow}(S \otimes P) \otimes Q \stackrel{\alpha_{S}}{\longrightarrow} S
$$

by $\theta$ we see that $\theta: P \otimes Q \rightarrow S$ is an isomorphism in ${ }_{S}$ Act $_{S}$. Similarly we obtain an isomorphism $\phi: Q \otimes P \rightarrow T$ in ${ }_{T}$ Act $_{T}$. An application of Lemma 5.8 gives us a unitary bijective Morita context.

$(3) \Rightarrow(4)$. This is obvious.

(4) $\Rightarrow(1)$. Assume that $S$ and $T$ are strongly Morita equivalent via a unitary surjective Morita context $\left(S, T,{ }_{S} P_{T},{ }_{T} Q_{S}, \theta, \phi\right)$. We know that the firm acts and the acts fixed by $\Gamma$ are the same (see Theorem 3.16). Hence, by the dual of Theorem 3 in [36], we can say that the funcors

$$
\begin{aligned}
& \operatorname{Act}_{S}(Q,-) T \otimes T: \mathrm{FAct}_{S} \rightarrow \mathrm{FAct}_{T}, \\
& \operatorname{Act}_{T}(P,-) S \otimes S: \mathrm{FAct}_{T} \rightarrow \mathrm{FAct}_{S}
\end{aligned}
$$

are mutually inverse equivalence functors.

$(4) \Leftrightarrow(5)$ This holds by Proposition 14 in [23].

$(1) \Leftrightarrow(2)$ This follows from the left-right symmetry of condition (3).

Remark 5.10. To prove Theorem 5.9 we did not use generators (not to speak about indecomposable projectives) at all. This is a remarkable difference from all proofs of similar results that we are aware of.

Still, generators are implicitly present. By Corollary $3.20, S_{S}$ is a generator in FAct $S_{S}$ and $T_{T}$ is a generator in FAct $T$, thus $P_{T}=F(S)$ and $Q_{S}=G(T)$ are generators, too. 


\section{REFERENCES}

[1] G. D. Abrams, Morita equivalence for rings with local units, Commun. Algebra 11 (1983), 801-837.

[2] J. Adámek, J. Rosický, Locally Presentable and Accessible Categories, Cambridge University Press, 1994.

[3] B. Afara, M. V. Lawson, Morita equivalence of semigroups with locally commuting idempotents, Commun. Algebr. 40 (2012), 1982-1996.

[4] B. Afara, M. V. Lawson, Morita equivalence of inverse semigroups, Period. Math. Hungar. 66 (2013), 119-130.

[5] P. N. Ánh, L. Márki, Morita equivalence for rings without identity, Tsukuba J. Math. 11 (1987), 1-16.

[6] B. Banaschewski, Functors into categories of $M$-sets, Abh. Math. Sem. Univ. Hamburg 8 (1972), 49-64.

[7] F. Borceux, Handbook of Categorical Algebra 1: Basic Category Theory, Cambridge University Press, Cambridge, 1994.

[8] F. Borceux, Handbook of Categorical Algebra 2: Categories and structures, Cambridge University Press, Cambridge, 1994.

[9] Y. Q. Chen, K. P. Shum, Morita equivalence for factorisable semigroups, Acta Math. Sin. (Engl. Ser.) 17 (2001), 437-454.

[10] E. Cheng, N. Gurski, E. Riehl, Cyclic multicategories, multivariable adjunctions and mates, J. K-Theory 13 (2014), 337-396.

[11] D. Dikranjan, W. Tholen, Categorical structure of closure operators, Mathematics and its Applications, 346. Kluwer Academic Publishers Group, Dordrecht, 1995.

[12] S. Eilenberg, J. C. Moore, Adjoint functors and triples, Illinois J. Math. 9 (1965), 381398.

[13] L. El Kaoutit, Wide Morita contexts in bicategories, Arab. J. Sci. Eng. 1320 (2008), 153-173.

[14] J. Funk, M. V. Lawson, B. Steinberg, Characterizations of Morita equivalence of inverse semigroups, J. Pure Appl. Algebra 215 (2011), 2262-2279.

[15] J. L. García, L. Marín, Rings having a Morita-like equivalence, Commun. Algebra 27 (1999), $665-680$

[16] J. L. García, L. Marín, Some properties of tensor-idempotent rings. In: Algebra and its applications (Athens, OH, 1999), 223-235, Contemp. Math., 259, Amer. Math. Soc., Providence, RI, 2000.

[17] J. L. García, J. J. Simón, Morita equivalence for idempotent rings, J. Pure Appl. Algebra 76 (1991), 39-56.

[18] J. González-Férez, L. Marín, Exactness of direct limits in the category of firm modules. J. Algebra 330 (2011), 298-313.

[19] G. M. Kelly, F. W. Lawvere, On the complete lattice of essential localizations, Bull. Soc. Math. Belg. Sr. A 41 (1989), 289-319.

[20] M. Kilp, U. Knauer, A. Mikhalev, Monoids, Acts and Categories, de Gruyter Expositions in Mathematics, vol. 29, Walter de Gruyter, Berlin, New York, 2000.

[21] U. Knauer, Projectivity of acts and Morita equivalence of monoids, Semigroup Forum 3 (1972), 359-370.

[22] V. Laan, Context equivalence of semigroups, Period. Math. Hungar. 60 (2010), 81-94.

[23] V. Laan, L. Márki, Strong Morita equivalence of semigroups with local units, J. Pure Appl. Algebra 215 (2011), 2538-2546.

[24] V. Laan, L. Márki, Morita invariants for semigroups with local units, Mh. Math. 166 (2012), $441-451$.

[25] V. Laan, L. Márki, Fair semigroups and Morita equivalence, Semigroup Forum 92 (2016), 633-644.

[26] J. Lambek, P. J. Scott, Introduction to higher order categorical logic. Cambridge University Press, 1988.

[27] M. V. Lawson, Morita equivalence of semigroups with local units, J. Pure Appl. Algebra 215 (2011), 455-470.

[28] L. Marín, Morita equivalence based on contexts for various categories of modules over associative rings. J. Pure Appl. Algebra 133 (1998), 219-232.

[29] V. V. Neklyudova, Polygons over semigroups with a system of local units, Fundam. Appl. Math. 3 (1997), 879-902 (in Russian). 
[30] V. V. Neklyudova, Morita equivalence of semigroups with a system of local units, Fundam. Appl. Math. 5 (1999), 539-555 (in Russian).

[31] D. Quillen, Module theory over nonunital rings, notes, 1996. Available at http://www.claymath.org/library/Quillen/Working-papers/quillen\%201996/1996-2.pdf

[32] D. Quillen, Morita equivalence for non-unital rings, unpublished notes from a lecture at the University of Exeter, 8.2.1996.

[33] S. K. Sardar, S. Gupta, A note on Morita invariants of semigroups, Semigroup Forum 92 (2016), 71-76.

[34] B. Steinberg, Strong Morita equivalence of inverse semigroups, Houston J. Math. 37 (2011), 895-927.

[35] S. Talwar, Morita equivalence for semigroups, J. Austral. Math. Soc. (Series A) 59 (1995), $81-111$.

[36] S. Talwar, Strong Morita equivalence and a generalisation of the Rees theorem, J. Algebra 181 (1996), 371-394.

[37] S. Talwar, Strong Morita equivalence and the synthesis theorem, Internat. J. Algebra Comput. 6 (1996), 123-141.

[38] J. L. Taylor, A bigger Brauer group, Pacific J. Math. 103 (1982), 163-203.

[39] Y. Xu, K. P. Shum, R. F. Turner-Smith, Morita-like equivalence of infinite matrix subrings, J. Algebra 159 (1993), 425-435.

Institute of Mathematics, Faculty of Mathematics and Computer Science, J. Litvi 2, University OF TARTU, 50409 TARTU, Estonia

E-mail address: vlaan@ut.ee

Alfréd Rényi Institute of Mathematics, Hungarian Academy of Sciences, H-1364 BuDAPEST, Pf. 127, Hungary

E-mail address: marki.laszlo@renyi.mta.hu

Institute of Mathematics, Faculty of Mathematics and Computer Science, J. Liivi 2, University of TARTu, 50409 Tartu, Estonia

E-mail address: ulo.reimaa@gmail.com 